\title{
BOUNDING BOX METHOD BASED ACCURATE VEHICLE NUMBER DETECTION AND RECOGNITION FOR HIGH SPEED APPLICATIONS
}

\author{
V. Baranidharan ${ }^{1}$, Kiruthiga Varadharajan ${ }^{2}$, K. Sudhakar ${ }^{3}$ and N.V. Lokesh $^{4}$ \\ ${ }^{1,3,4}$ Department of Electronics and Communication Engineering, Bannari Amman Institute of Technology, India \\ ${ }^{2}$ Department of Computer and Information Sciences, Annamalai University, India
}

\begin{abstract}
License plate detection and recognition is the one of the major aspects of applying the image processing techniques towards intelligent transport systems. Detecting the exact location of the license plate from the vehicle image at very high speed is the one of the most crucial step for vehicle plate detection systems. This paper proposes an algorithm to detect license plate region and edge processing both vertically and horizontally to improve the performance of the systems for high speed applications. Throughout the detection and recognition the original images are detected, filtered both vertically and horizontally, and threshold based on bounding box method. The whole system was tested on more than twenty five cars with various license plates in Indian style at different weather conditions. The overall accuracy rate of success recognition is $93 \%$ at sunlight conditions, $72 \%$ at cloudy, $71 \%$ at shaded weather conditions.
\end{abstract}

Keywords:

Plate Detection, Recognition, Segmentation, Noise Removal, Sobel Detector, Bounding Box

\section{INTRODUCTION}

Now days, the high speed vehicles are plays a vital role in transportation. The usage of high speed vehicles are also increase exponentially due to the rapid growth of the population and its needs. This high speed vehicles are having a vast and variety of applications, includes travel time estimation, highway car counting's, traffic violations detections, and surveillance applications.

The license plate (Vehicle plate) recognition and character recognition is an image processing techniques to recognize the authorized vehicles, speed of the vehicles by tracking the license plate by using mounted cameras in the traffic signals by capturing the images. These cameras are having very high resolutions to take an image that starts from 20 to 50 more meters away from the camera locations. This high resolutions camera installation point causes many difficulties against accurate detection of license plate and the recognition characters in that plates. In India, there are only two types of number plates are widely used. They are,

- Black letters with white background on the plates.

- Black letters with yellow background on the plates.

In many of the countries, the violation of the colours is also as evidence. For such evidence purposes, the colour cameras are also located in point of the traffic signals. These colour cameras are placed in the tunnels for controlled lighting conditions. This paper focuses on the evaluation of systems for two industrial applications, is to find the traffic violations and to counting the number of vehicles in the highways.
The above two applications are very important in the Intelligent Transport Systems (ITS) industry for taking a further actions against the high speed vehicles. The vehicle plate recognition is divided into a very important three main parts. They are Plate detection, Character segmentation and Character recognition. The above three process may have a problems when plate size variations, viewing angle variations, low contrast plates, high speed vehicles, time consuming recognition algorithms and dirty/filthy vehicles plates.

The paper is organized as follows: in section 2 explains the literature survey. In section 3 discuss methodology and algorithm for vehicle plate detection and character recognition. Section 4 explains the simulated results and conclusion is made in section 5 .

\section{LITERATURE SURVEY}

Vehicle license plate detection and character recognition are very important in secured transport systems for high speed applications. Most of the researchers had used different techniques and methods to detect the recognition of vehicle number plate in various journals, books, transactions, and conference papers. The information is collected in the relevant field from previous works. There are some fundamental processing steps: Image enhancement, Number plate detection from the image, Character segmentation and Character recognition. For license plate detection, there are several algorithms have been proposed. Some of these algorithms are analysed below.

Mousa [1] proposes plate detection algorithms are localized using canny edge detector. Sobel operators are only used for detecting image edges. These methods are having two advantages: Smoothing the noise in an image because it includes averaging, generating thick and bright edges for differentiating rows and columns. The main advantages are low computational complexity and memory requirements.

Du [2] suggests Automatic License Plate Recognition (ALPR) algorithm using Hough transforms. Hough transforms are used to detect license plate by finding the borders of the plates. This transforms is a memory and time consuming process. In this wavelet based methods, high frequency coefficients are used to detect an accuracy of the plates. This algorithm fails on gray scale images or an image with low colour disparity.

Ghofrani [3] proposed vehicle plate detection algorithm based on the Mathematical Morphology and Connected Component analysis. These algorithms are first enhancing contrast of the plates and apply detection algorithms. These algorithms are very successful in identifying the clean plates but it not very suitable for dirty images. 
Sarfraz [4] proposed Morphological operations based connected components analysis. These algorithms use a proper thresholding method to obtain the binary image of the plate before the further pre-processing. This detection step is not having input plate quality and parameters are cannot be tuned appropriately. Therefore, the recognition accuracy of these algorithms decreases when the plate qualities are involved.

In some methods, the character recognition is based on key points of localization like SIFT and SURF algorithms [5] [6]. The major disadvantages of these algorithms are taking more times and very high complexity [7]. We have proposed a new feature set for character recognition features are based on bounding box of our systems. The final systems are capable for detecting and recognizing of multiple license plates in a single frame of high speed applications.

\section{METHODOLOGY OF LICENSE PLATE DETECTION}

The main goal of this section is to explain the methods of finding the high speed vehicles plate location in captured images. The High definition camera is always a monochrome camera with infrared projector and colour camera are employed in Intelligent Transport systems with ANPR systems [9] [10]. These projectors are coated with IR reflection materials. These IR projectors are very important in detecting dirty/filthy plates even in daylight and night time. IR projector power has a very close relation with camera exposure time and plays with a final clarity of the vehicle plates. Modifying the exposure time based on lightning conditions is performed in an adaptive procedure that gets its feedback from the thickness of license plates. The general block diagram of vehicle number plate detection and recognition systems are shown in the Fig.1 [11] [12]. There are four main steps and have a vital role in this process.

- Pre-processing

- Localization of license plate region

- Character segmentation of license plate

- Character recognition of license number plate using bounding box method for high speed vehicles.

\subsection{PRE-PROCESSING}

The images of the vehicles are captured using High Definition camera of 13 Mega-pixel resolution cameras, whose number plates are to be identified. Images are selecting from the frame depending upon the video length and time taken for which video is clear. To selecting the frame can be done by using the below Eq.(1),

$$
\text { Required Frame }(F)=(N \times t) / T
$$

where, $F$ is the required image or frame from the video, $N$ is the total number of frames, $t$ is the time required and $T$ is the total time of video or total length of the video. The image size is used as $1200 \times 1600$.

The pre-processing step is need to convert the colour RGB images to Gray scale images using the Eq.(2),

$$
I=[(0.114 \times R)+(0.587 \times G)+(0.299 \times B)]
$$

In order, to enhance the number plate recognition we use the filter to eliminate the noise but it also very concentrates in high frequency images. Edge detection is very important in this algorithm for vehicle plate detection and character recognition. The license plate should be in rectangular in shape.

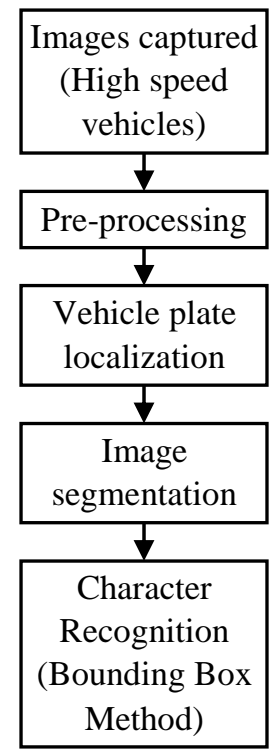

Fig.1. General System Block Diagram

\subsection{LOCALIZATION OF THE VEHICLE PLATE}

The ultimate aim is to identify the vehicle plate from the captured image. This can be achieved by two important steps, they are,

- Finding exact location of number plates from the captured images.

- Location the large bounding using license plate.

The main advantages are the vehicle number plates are having very high contrast areas with yellow and white background and letters are in black colour. The intensity is also varies horizontally but the characters are placed vertically. The boundaries of an image are identified by using sobel operator. This filter identifies the edges based on the sharp variation of the intensity gradient value of an image [13]. Edges are having a very high intensity contrast from each and every pixel. The edges identification is plays a vital role in removing the unnecessary information.

The gradient calculation from the images is calculated by using the below equation,

$$
\nabla f=\text { Magnitude }(\nabla f)=\left\{G_{x}+G_{y}\right\}^{0.5}
$$

Where, $\nabla f$ - Magnitude vector, $G_{x}, G_{y}$ - Direction in $x$ and $y$. In the Eq.(4) and Eq.(5) explains the sobel mask/ matrix is also considered for both direction $x$ and $y$.

For $x$ direction,

$$
\left[\begin{array}{ccc}
-1 & -2 & -1 \\
0 & 0 & 0 \\
1 & 2 & 1
\end{array}\right]
$$

For $y$ direction,

$$
\left[\begin{array}{lll}
-1 & 0 & 1 \\
-2 & 0 & 2 \\
-1 & 0 & 1
\end{array}\right]
$$




\subsection{CHARACTER SEGMENTATION OF THE NUMBER PLATE}

The number plates regions are extracted from the captured image. The extracted image will scans with the connected components are identified and it will label it. Each connected components are differentiated with each other. The number plates are divided into multiple numbers of sub images. Each sub images are having at least one character in it. After segmentation process, bounding box method is used to compare the sub image with predefined templates. After making the comparison, the connected regions are labelled; it determines the coordinate's corner of the bounding box and its width and heights. The paper filled bounding box is used to relaying the covering the connected objects and also measures the image region properties.

\subsection{CHARACTER RECOGNITION OF THE NUMBER PLATE}

The next step is character recognition after image segmentation. The main aim of the recognition of the extract the characters from the image. Each of the characters are compared with alphanumeric database which uses templates for matching. In matching process, the sub-images are compared with template image in the possible position in the database. The numeric index will be calculated for all the sub-images. This template matching is the process of finding the exact location of the sub-image inside the template images. To find the similarity in between the objects and template images by using normalized cross correlation equations is given below,

$$
N_{c c}\left(I_{1}, I_{2}\right)=\frac{1}{N_{\sigma_{1} \sigma_{2}}} \sum_{x, y=0}^{n} I(x, y)
$$

where, $I_{1}$ - Template Image, $I_{2}$ - Input image and $N_{c c}$ - Normalized Cross Correlation value.

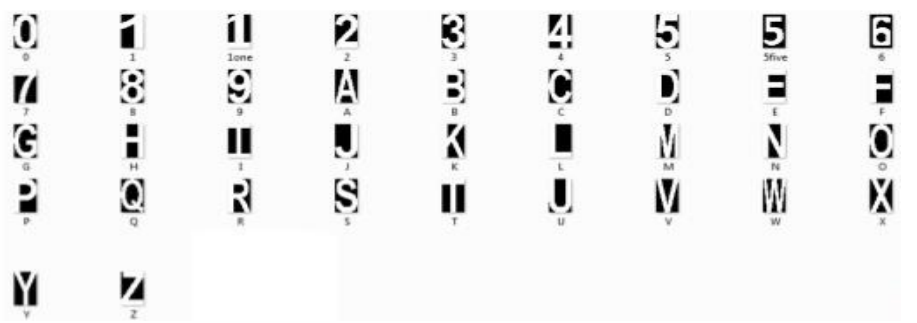

Fig.2. Template for character recognition

The obtained cross correlation value is higher than the threshold value than it is considered as a new one. The score are matched with the existing template images, if it score more values than the previous threshold value then it will automatically updated with the higher degree. The suitable matched characters values are recovered and the output is stored in a text file. The size of this output image is always in $42 \times 42$ pixels as shown in the Fig.2.

\section{SIMULATION RESULTS}

The experiment was conducted on Matrix Laboratory (MATLAB) R2017a simulator used for digital image processing and analysis.

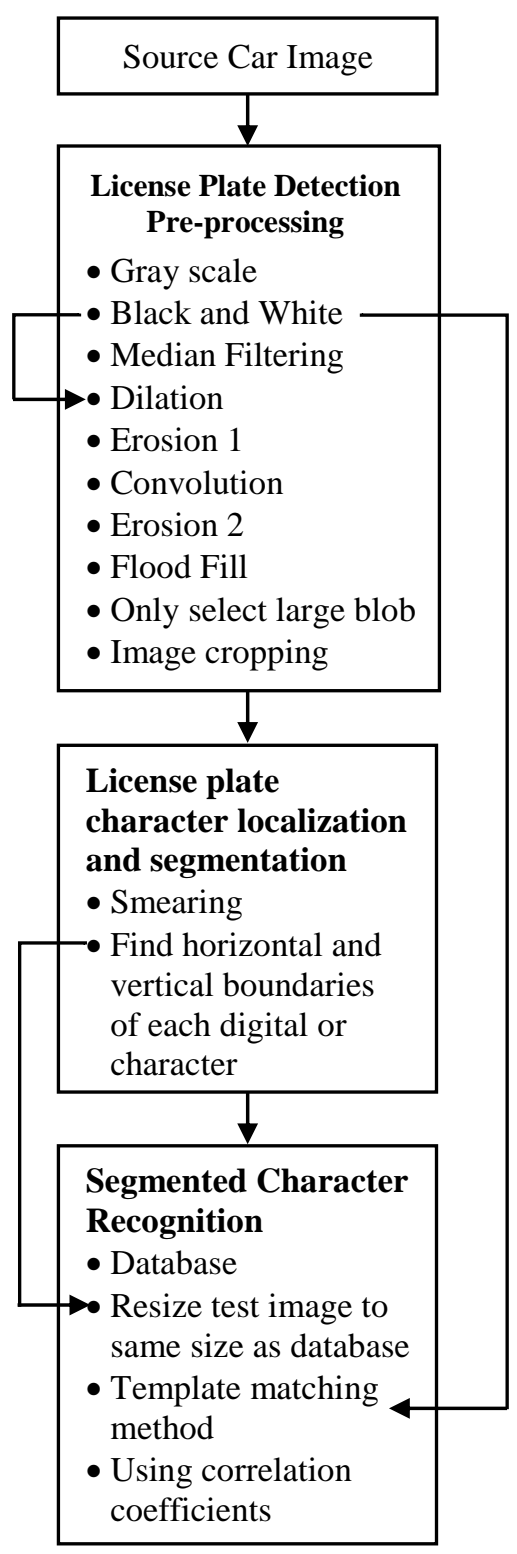

Fig.3. Proposed system

The vehicle license plate detection and recognition algorithms is given as following steps,

Step 1: Captured Images: The images are captured by using High Definition (HD Quality) cameras and the images are given as an input to process.

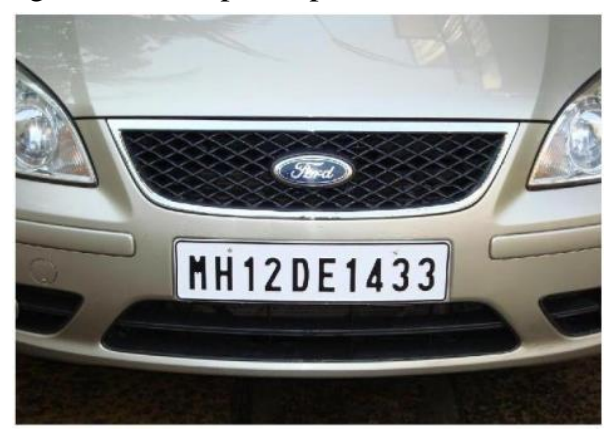

Fig.4. Captured Image using HD Cameras 
Step 2: Gray Scale conversion: The colour images are converted into a gray scale image using the above mentioned Eq.(1)-Eq.(5).

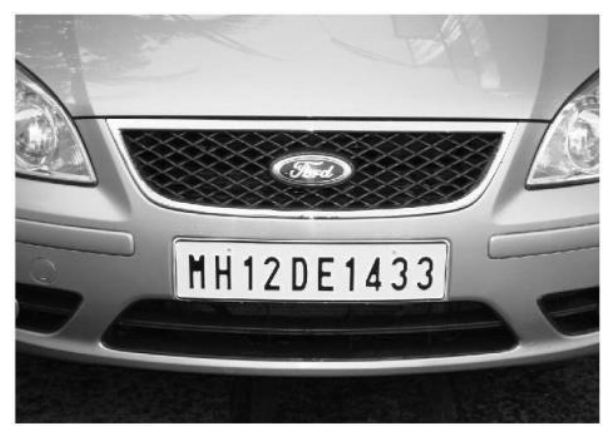

Fig.5. Gray Scale Converted Image

Step 3: Image enhancement: Unwanted noises of the images are removed by using filters.

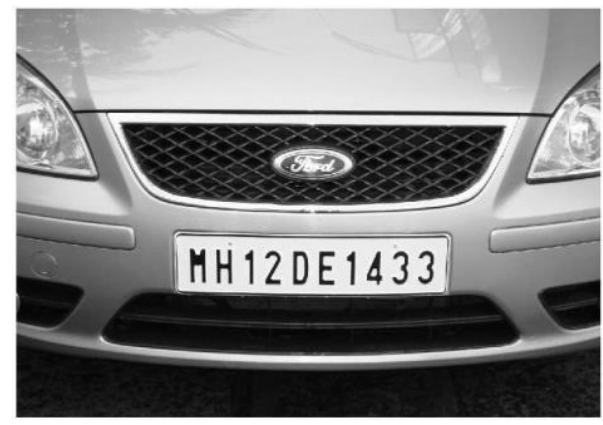

Fig.6. Image enhancement

Step 4: Vehicle plate extraction: the rows and column values are identified from the image to identify the plate region. Sobel edge detector is used to analyse the boundaries then it will be dilated image and remove the unwanted connected objects from the image.
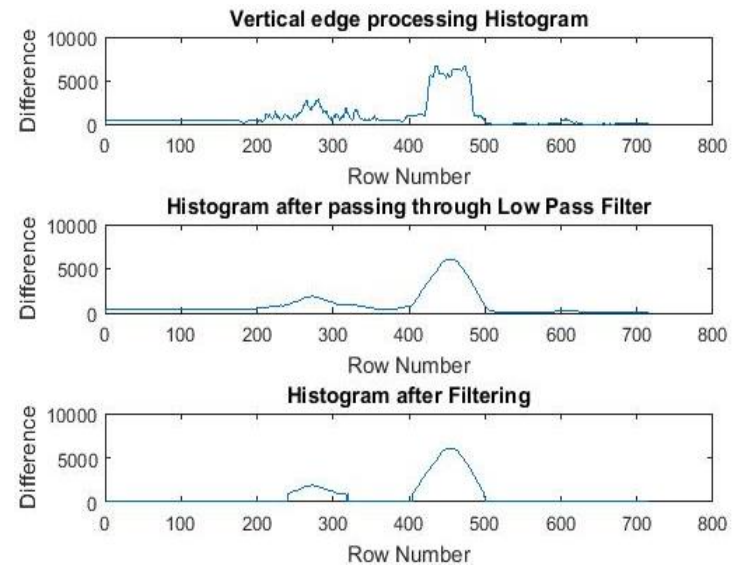

Fig.7. Vehicle plate extraction using Sobel edge detector (Vertical Processing)
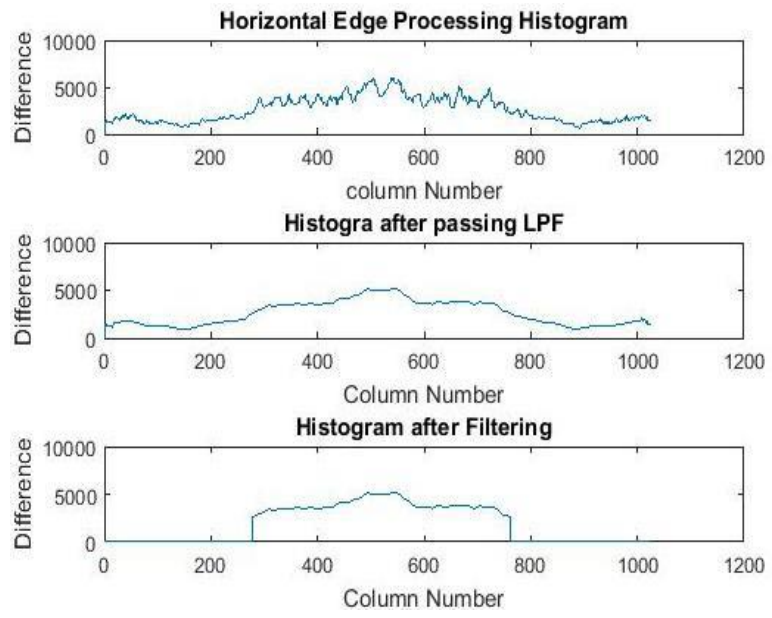

Fig.8. Vehicle plate extraction using Sobel edge detector (Horizontal Processing)

Step 5: Character Segmentation and Character recognition: the bounding box method is used to map the each character, for each letter it mapped with a box values and displayed the each character into a single images as shown in the Fig.9. After segmenting the each character from the templates, the characters are compared with the each letters pixel values. If it exceeds the threshold value, then the pixels corresponding to the images are found and it will be displayed in the text.

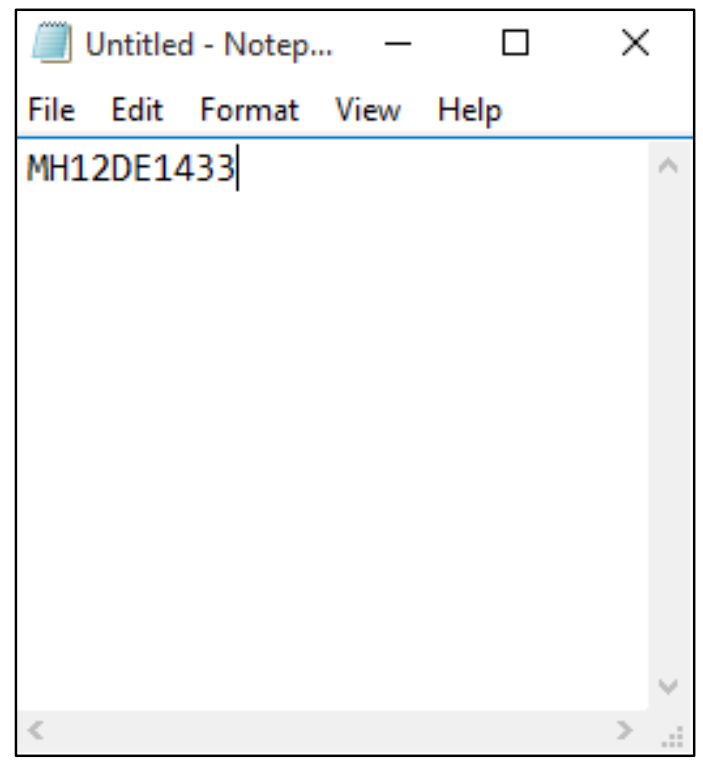

Fig.9. Plate number extraction using bounding box method

The Table. 1 reports the evaluation of the proposed system over existing systems. According to this table, the proposed system outperforms than the existing systems based on the data sets. In this evaluation, the overall accuracy is based on the given equation,

$$
\text { Overall Accuracy }=(D \times S \times R) \%
$$

where, $D$ - Detected license plate region, $S$ - Segmentation rate for the characters and $\mathrm{R}$ - Recognition rate. Here, the detection rate is defined as the number of the detected plates divided by the total presented plates. The character segmentation rate is equals to the 
ratio of the number of the correctly segmented characters to the total number of the characters in the set. The character recognition plate is the proportion of the correctly classified characters over the data set.

Table.1. Evaluation of the proposed systems and the comparison with the existing Reported systems

\begin{tabular}{|c|c|c|c|c|c|c|}
\hline \multirow{2}{*}{$\begin{array}{c}\text { License } \\
\text { plate } \\
\text { Weather } \\
\text { Conditions }\end{array}$} & \multicolumn{2}{|c|}{$\begin{array}{c}\text { Plate Detection } \\
\text { Accuracy }\end{array}$} & \multicolumn{2}{|c|}{$\begin{array}{c}\text { Plate Recognition } \\
\text { accuracy }\end{array}$} & \multicolumn{2}{|c|}{ Overall Accuracy } \\
\cline { 2 - 7 } & $\begin{array}{c}\text { Existing } \\
\text { System }\end{array}$ & $\begin{array}{c}\text { Proposed } \\
\text { System }\end{array}$ & $\begin{array}{c}\text { Existing } \\
\text { System }\end{array}$ & $\begin{array}{c}\text { Proposed } \\
\text { System }\end{array}$ & $\begin{array}{c}\text { Existing } \\
\text { System }\end{array}$ & $\begin{array}{c}\text { Proposed } \\
\text { System }\end{array}$ \\
\hline Sunlight & 90.36 & 100 & 90.86 & 100 & 90.6 & 100 \\
\hline $\begin{array}{c}\text { Cloudy } \\
\text { Weather }\end{array}$ & 62.66 & 71.55 & 61.8 & 75.5 & 61 & 72.8 \\
\hline Shade & 56.23 & 78.45 & 52.34 & 75.45 & 54.34 & 71.5 \\
\hline
\end{tabular}

It is worth noting that, the existing systems are not having the good accuracy at cloudy weather and shade weather conditions. $\mathrm{Bu}$ the proposed systems is comparatively good than the existing systems. This proposed algorithm is comparatively high accuracy in both plate segmentation and plate detection accuracy. Thus, the result of reference is depicted here for comparison.

\section{CONCLUSIONS}

The proposed systems are designed for the detection of the licensed plate and character recognition for high speed applications. At first, the capture the image, noise is removed, extract the interested number plate area using Sobel detector for both vertical and horizontal edges, and then segmented images are compared with pre-defined set of data sets using bounding box. The characters are recognized by using the cross correlation of the processed image with the templates matching. In future work, the processing of multiple images of vehicle plates are processed parallel at a time. This proposed system will shed to detect the license plate of vehicles in high speed applications.

\section{REFERENCES}

[1] A. Mousa, "Canny Edge-Detection based Vehicle Plate Recognition”, International Journal of Signal Processing, Image Processing and Pattern Recognition, Vol. 5, No. 3, pp. 1-8, 2012.
[2] S. Du, M. Ibrahim, M. Shehata and W. Badawy, "Automatic License Plate Recognition (ALPR): A State-of-the-Art Review", IEEE Transactions on Circuits and Systems for Video Technology, Vol. 23, No. 2, pp. 311-325, 2013.

[3] S. Ghofrani and M. Rasouli, "Farsi License Plate Detection and Recognition based on Characters Features", Majlesi Journal of Electrical Engineering, Vol. 5, No. 2, pp. 44-51, 2011.

[4] M.S. Sarfraz, A. Shahzad, M.A. Elahi, M. Fraz, I. Zafar and E.A. Edirisinghe, "Real-Time Automatic License Plate Recognition for CCTV Forensic Applications", Journal of Real-Time Image Processing, Vol. 8, No. 3, pp. 285-295, 2013.

[5] R. Azad, F. Davami and B. Azad, "A Novel and Robust Method for Automatic License Plate Recognition System based on Pattern Recognition", International Journal of Advanced Computer Science and Applications, Vol. 2, No. 3, pp. 64-70, 2013.

[6] H. Caner, H.S. Gecim and A.Z. Alkar, "Efficient Embedded Neural Network-based License Plate Recognition System", IEEE Transactions on Vehicular Technology, Vol. 57, No. 5, pp. 2675-2683, 2008

[7] S.D. Palmer and O.N. Aharoni, "System for Collision Prediction and Traffic Violation Detection," U.S. Patent 20 130093 895, Apr. 18, 2013.

[8] G. Liu, Z. Ma, Z. Du and C. Wen, "The Calculation Method of Road Travel Time based on License Plate Recognition Technology", Proceedings of International Conference on Computer Science and Education, pp. 385-389, 2011,

[9] J.M. Guo and Y.F. Liu, "License Plate Localization and Character Segmentation with Feedback Self-Learning and Hybrid Binarization Techniques", IEEE Transactions on Vehicular Technology, Vol. 57, No. 3, pp. 1417-1424, 2008.

[10] P.H. Torr and D.W. Murray, "The Development and Comparison of Robust Methods for Estimating the Fundamental Matrix", International Journal of Computer Vision, Vol. 24, No. 3, pp. 271-300, 1997.

[11] Y.W. Chen and C.J. Lin, "Combining SVMs with Various Feature Selection Strategies", Available at: https://www.csie.ntu.edu.tw/ cjlin/papers/features.pdf.

[12] J. Weston, S. Barnhill and V. Vapnik, "Gene Selection for Cancer Classification using Support Vector Machines," Machine Learning, Vol. 46, No. 1-3, pp. 389-422, 2002.

[13] S. Wold, K. Esbensen and P. Geladi, "Principal Component Analysis", Chemometrics and Intelligent Laboratory Systems, Vol. 2, No. 1, pp. 37-52, 1987. 